\title{
Structural Relaxation Monitored by Instantaneous Shear Modulus
}

\author{
Niels Boye Olsen, Jeppe C. Dyre, and Tage Christensen \\ Department of Mathematics and Physics (IMFUFA), Roskilde University, P.O. Box 260, DK-4000 Roskilde, Denmark
}

(Received 14 January 1998)

This paper reports on aging of the silicone oil MS704 for sudden changes of temperature from 210.5 to $209.0 \mathrm{~K}$ and from 207.5 to $209.0 \mathrm{~K}$ studied by continuously monitoring the instantaneous shear modulus $G_{\infty}$. The results are interpreted within the Tool-Narayanaswamy formalism with a reduced time definition based on a recently proposed expression for the relaxation time, where $G_{\infty}$ reflects the fictive temperature. All parameters entering the reduced time were determined from independent measurements of the frequency-dependent shear modulus of the equilibrium liquid. [S0031-9007(98)06756-8]

PACS numbers: 64.70.Pf, 62.80. $+\mathrm{f}$

Viscous liquids and the glass transition are old research fields, which continue to attract attention because of major unsolved problems [1-11]. One problem is the almost ubiquitous non-Arrhenius average relaxation times. With few exceptions, average relaxation times of highly viscous liquids increase more dramatically upon cooling than expected from an Arrhenius law. The viscosity $\eta$ is also non-Arrhenius, reflecting the fact that $\eta$ is related to the average shear relaxation time $\tau$ by Maxwell's expression

$$
\tau=\frac{\eta}{G_{\infty}} .
$$

Here $G_{\infty}$ is the instantaneous (infinite-frequency) shear modulus, a quantity that is only moderately temperature dependent (usually increasing less than a factor of 4 upon cooling in the temperature range, where $\tau$ increases by 10 orders of magnitude). For typical cooling rates $\tau$ is of order $10^{3} \mathrm{~s}$ at the glass transition.

Many models have been proposed for the nonArrhenius average relaxation times of viscous liquids. However, only a few models are phenomenological in the sense that they relate $\tau(T)$ to other macroscopically measurable quantities. Well-known phenomenological models are the free-volume model of Cohen, Turnbull, and Grest [12-14] and the entropy model of Gibbs, DiMarzio, and Adam [15,16]. Briefly, the ideas behind these models are the following. In the free-volume model the molecules are modeled as hard spheres. A molecule is mostly confined to a cage bounded by its immediate neighbors. Occasionally, there is a fluctuation in density which opens up a hole within the cage large enough to permit a considerable displacement of the molecule. Molecular transport occurs only when a void having a "free" volume greater than some critical value forms. The dramatic non-Arrhenius increase of $\tau$ upon cooling is due to thermal contraction. In the entropy model the variable controlling the average relaxation time is the configurational entropy, the entropy in excess of the crystal entropy. It is well known [1] that this quantity extrapolates to zero at a nonzero temperature $T_{K}$ (the Kauzmann paradox). The basic idea of the entropy model is that close to $T_{K}$ the energy barrier restricting transitions between different molecular configurations is very high, because the few states that could conceivably occur are widely separated in phase space, so proceeding from one to another involves a considerable change in the topology of molecular entanglements. This gives rise to non-Arrhenius behavior because the barrier grows as the configurational entropy decreases upon cooling [16].

Recently, a phenomenological model in which $G_{\infty}$ controls the non-Arrhenius average relaxation time in viscous liquids was proposed [17]. It is assumed that the energy barrier has two contributions, one from the rearranging molecules themselves and one from "shoving" aside the surrounding liquid to reduce the first contribution. Anharmonicity of the intermolecular potential-with harsh repulsions but only weak attractions-implies that the shoving work gives the dominant contribution to the activation energy $[17,18]$. The shoving work is proportional to $G_{\infty}[17,18]$, leading to the following expression for the average relaxation time (where $V_{c}$ is a temperatureindependent volume):

$$
\tau=\tau_{0} \exp \left[\frac{G_{\infty} V_{c}}{k_{B} T}\right] .
$$

Non-Arrhenius behavior arises because $G_{\infty}$ increases as the temperature decreases. Equation (2), which was first discussed by Nemilov in 1968 in a different context [19] and recently also derived by Buchenau [20], is able to account for the temperature dependence of the average relaxation times of a number of glass-forming liquids $[17,20]$.

Viscosity or average relaxation time is probed by linear experiments, i.e., experiments where the liquid, in principle, is perturbed only infinitesimally from the equilibrium state. It is generally believed that structural relaxation, which is inherently nonlinear, and viscous flow are caused by the same basic molecular process. Therefore, if correct, Eq. (2) should also be applicable to structural relaxation experiments. Below, we present preliminary results for two such experiments, where relaxation following sudden temperature jumps — "aging"—is probed by continuously monitoring $G_{\infty}$. Similar experiments probing nonlinear relaxation by measuring high-frequency 
moduli by a different method (Brillouin spectroscopy) have been carried out by several groups [21-23], but our method gives less noisy data.

The measurements were done on the diffusion pump silicone oil MS704. This liquid has little dispersion at the resonance frequency of our experimental setup in the temperature range studied. The silicone oil was embedded in the one-disk version of the piezoelectric shear modulus gauge transducer (PSG) described elsewhere [24]. Temperature may be kept constant within $5 \mathrm{mK}$ over several days with an absolute uncertainty below $0.2 \mathrm{~K}$. The time constant for the temperature control of the sample is $400 \mathrm{~s}$.

The silicone oil was subjected to two sudden temperature jumps applied to well-annealed states of the sample. The first experiment involved a sudden change of temperature from 207.5 to $209.0 \mathrm{~K}$ ("up-jump"), the second involved a sudden temperature change from 210.5 to $209.0 \mathrm{~K}$ ("down-jump"). During the approach to equilibrium, $G_{\infty}$ was monitored by measuring the first resonance frequency $\omega_{0}$ of the PSG. Assuming that there are no significant shear relaxations above $\omega_{0}, G_{\infty}$ is given [24] by

$$
G_{\infty}=\rho d h\left(\omega_{0}^{2}-\Omega_{0}^{2}\right),
$$

where $\rho$ is the density of the piezoceramic, $d$ is the thickness of the liquid layer, $h$ is the thickness of the piezoceramic disk, and $\Omega_{0}$ is the resonance frequency of the empty axially clamped transducer. $\Omega_{0}$ was determined from a calibration of the transducer at $212 \mathrm{~K}$ by comparing the instantaneous shear modulus obtained by the resonance method with that obtained by the quasistatic method using the three-disk version [24] of the PSG. Typical values of $\omega_{0}$ are about $1.3 \times 10^{6} \mathrm{rad} / \mathrm{s}$. The high- $Q$ factor of the resonance peak (about 200) makes accurate measurements of the resonance frequency possible. The estimated relative uncertainty of resonance frequency is $3 \mathrm{ppm}$, which is better than the relative variation due to the $5 \mathrm{mK}$ temperature instability.

Figure 1 shows our data for up-jump and down-jump. As usual [3,25-27] the down-jump proceeds faster than the up-jump. This is because the "fictive temperature" $T_{f}$ [3,6,25-29], which controls the structural relaxation time is higher for the down-jump than for the up-jump. Asymptotically, of course, the fictive temperature in both cases converges to $209.0 \mathrm{~K}$.

We consider the simplest possible model consistent with Eq. (2). If $G_{\infty, \text { eq }}$ is the equilibrium value of $G_{\infty}$, this model follows Tool [28] in assuming that relaxation towards equilibrium is governed by the following simple first-order differential equation:

$$
\frac{d}{d t}\left(G_{\infty}-G_{\infty, \mathrm{eq}}\right)=-\frac{G_{\infty}-G_{\infty, \mathrm{eq}}}{\tau\left(G_{\infty}\right)} .
$$

In terms of the dimensionless variables $x=V_{c}\left(G_{\infty}-\right.$ $\left.G_{\infty, \text { eq }}\right) /\left(k_{B} T\right)$ and $\tilde{t}=t /\left\{\tau_{0} \exp \left[V_{c} G_{\infty, \text { eq }} /\left(k_{B} T\right)\right]\right\}$, Eq. (4) becomes $d x / d \tilde{t}=-x / \exp (x)$. If $\operatorname{Ei}(x)$ denotes the

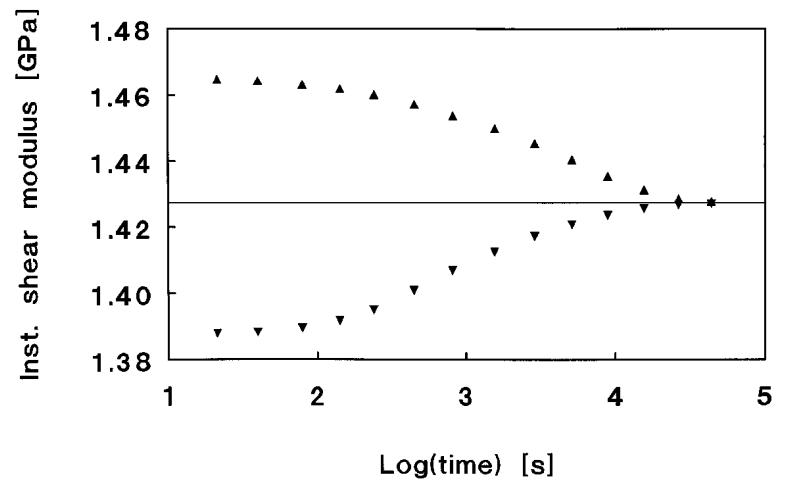

FIG. 1. Instantaneous shear modulus $G_{\infty}$ for the silicone oil MS704 as function of time after sudden temperature jumps from 207.5 to $209.0 \mathrm{~K}$ (up triangles) and from 210.5 to $209.0 \mathrm{~K}$ (down triangles). The horizontal line marks the equilibrium value of $G_{\infty}$ at $209.0 \mathrm{~K}$. As usual, in structural relaxation, the relaxation towards equilibrium proceeds faster for downjump than for up-jump. $G_{\infty}$ was monitored by means of the piezoelectric shear modulus gauge transducer in the resonance mode [24]. This method measures the shear modulus $G(\omega)$ at about $\omega=1.3 \times 10^{6} \mathrm{rad} / \mathrm{s}$ [the identification of $G(\omega)$ with $G_{\infty}$ involves the assumption that there are no significant shear losses at higher frequencies].

exponential integral characterized by $\frac{d}{d x} E i(x)=$ $\exp (x) / x$, the solution is

$$
\tilde{t}=\operatorname{Ei}(x(0))-\operatorname{Ei}(x(\tilde{t})) .
$$

Our experiments, however, are poorly fitted by Eq. (5). The reason is the well-known fact that a distribution of relaxation times is always involved in structural relaxation. This is reflected in memory effects as seen in, e.g., the overshoot phenomenon [26].

A distribution of relaxation times is allowed for in the Tool-Narayanaswamy (TN) formalism [3,25-35]. For a sudden temperature jump at $t=0$ the TN formalism predicts that the normalized relaxation function is a function solely of the "reduced time" that has passed since $t=0$. The reduced time increment is the time increment measured in units of the relaxation time (the latter quantity itself relaxing with time). The two most commonly used relaxation time formulas are the Narayanaswamy-Moynihan [30,31] and the Adam-Gibbs expressions $[16,32,35]$. Based on Eq. (2) for the relaxation time, where $G_{\infty}$ itself is a measure of the fictive temperature, we here define the reduced time $\xi$ by

$$
\xi=\int_{0}^{t} \frac{d t^{\prime}}{\tau\left(G_{\infty}\left(t^{\prime}\right)\right)} .
$$

According to the TN formalism, both relaxation experiments are-for some suitable function $F(\xi)$-described by

$$
\frac{G_{\infty}(t)-G_{\infty, \mathrm{eq}}}{G_{\infty}(0)-G_{\infty, \mathrm{eq}}}=F(\xi) .
$$

In Eq. (7) the glassy (instantaneous) change of $G_{\infty}$ is not subtracted as usually done, because, assuming that both the glassy change and the overall change of $G_{\infty}$ are 
proportional to the temperature change, this is not necessary when the two temperature jumps have equal magnitude. Note that the simple model of Eq. (4) corresponds to $F(\xi)=\exp (-\xi)$ and no glassy change of $G_{\infty}$.

Figure 2 shows the normalized relaxation function plotted as a function of $\xi$ for both up- and down-jump on a linear time scale (used because, due to the long thermal time constant of the setup, only in the last 1-1.5 decade of the measurements of Fig. 1 is the temperature equilibrated). The two curves coincide, showing that structural relaxation of $G_{\infty}$ is consistently described by the TN formalism in conjunction with Eq. (2). The parameters $\tau_{0}$ and $V_{c}$ of Eq. (2) were determined from measurements of $G_{\infty}$ and the shear mechanical loss peak frequency in the equilibrium liquid state, both linear experiments. These measurements were carried out at 212 and $214 \mathrm{~K}$ (the lowest temperatures possible), leading to the following parameters of Eq. (2): $V_{c}=81 \AA^{3}$ and $\tau_{0}=8 \times 10^{-16} \mathrm{~s}$.

In the present experiment, only relatively small temperature jumps were studied, and the overall change of $G_{\infty}$ is just 5\%. However, this is much larger than the changes of other directly measurable quantities modeled by the TN formalism such as volume [36] or refractive index [37]. Also, it should be noted that for the liquid studied the $5 \%$ change of $G_{\infty}$ corresponds to more than 1 order of magnitude change of the average relaxation time, thus ensuring that the experiment is far from linearity.

In conclusion, we have shown that aging of the instantaneous shear modulus in MS704 may be described by the $\mathrm{TN}$ formalism in conjunction with the reduced time definition of Eq. (6). This, however, does not provide a definitive test of Eq. (2). Such a test would involve showing that the above approach works better

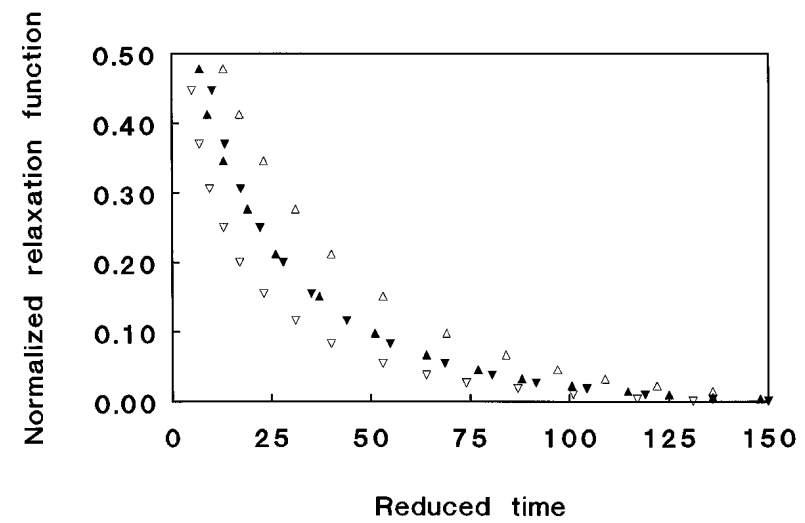

FIG. 2. Normalized relaxation function for the two temperature jumps of Fig. 1 plotted as a function of the reduced time defined in Eq. (6) (solid symbols) on a linear time scale. The open symbols give the "raw" normalized relaxation function as a function of a dimensionless time defined by the overall scaling of the actual time with the equilibrium average relaxation time at $209.0 \mathrm{~K}$. The fact that the two curves of solid symbols coincide shows that the Tool-Narayanaswamy formalism with the reduced time definition given in Eq. (6) works well for the aging of MS704. than the TN formalism with either the NarayanaswamyMoynihan [30,31] or the Adam-Gibbs [16,32,35] reduced time definitions.

This work was supported by the Danish Natural Science Research Council.

[1] W. Kauzmann, Chem. Rev. 43, 219 (1948).

[2] G. Harrison, The Dynamic Properties of Supercooled Liquids (Academic Press, New York, 1976).

[3] S. Brawer, Relaxation in Viscous Liquids and Glasses (American Ceramic Society, Columbus, OH, 1985).

[4] J. Jäckle, Rep. Prog. Phys. 49, 171 (1986).

[5] C. A. Angell, J. Phys. Chem. Solids 49, 863 (1988).

[6] G. W. Scherer, J. Non-Cryst. Solids 123, 75 (1990).

[7] C. A. Angell, J. Non-Cryst. Solids 131-133, 13 (1991).

[8] A. Hunt, J. Non-Cryst. Solids 160, 183 (1993).

[9] C. A. Angell, Science 267, 1924 (1995).

[10] U. Mohanty, Adv. Chem. Phys. 89, 89 (1995).

[11] M.D. Ediger, C.A. Angell, and S.R. Nagel, J. Phys. Chem. 100, 13200 (1996).

[12] M.H. Cohen and D. Turnbull, J. Chem. Phys. 31, 1164 (1959).

[13] D. Turnbull and M.H. Cohen, J. Chem. Phys. 34, 120 (1961).

[14] G. S. Grest and M. H. Cohen, Adv. Chem. Phys. 48, 455 (1981).

[15] J.H. Gibbs and E. A. DiMarzio, J. Chem. Phys. 28, 373 (1958).

[16] G. Adam and J. H. Gibbs, J. Chem. Phys. 43, 139 (1965).

[17] J. C. Dyre, N. B. Olsen, and T. Christensen, Phys. Rev. B 53, 2171 (1996).

[18] J. C. Dyre, J. Non-Cryst. Solids (to be published).

[19] S. V. Nemilov, Russ. J. Phys. Chem. 42, 726 (1968).

[20] U. Buchenau, J. Non-Cryst. Solids (to be published).

[21] J. K. Krüger, R. Roberts, H.-G. Unruh, K.-P. Frühauf, J. Helwig, and H. E. Müser, Prog. Colloid Polym. Sci. 71, 77 (1985).

[22] P. Bezot and C. Hesse-Bezot, J. Non-Cryst. Solids 122, 160 (1990).

[23] R. S. Miller and R. A. MacPhail, J. Chem. Phys. 106, 3393 (1997).

[24] T. Christensen and N. B. Olsen, Rev. Sci. Instrum. 66, 5019 (1995).

[25] O. V. Mazurin, J. Non-Cryst. Solids 25, 130 (1977).

[26] G.W. Scherer, Relaxation in Glass and Composites (Wiley, New York, 1986).

[27] G. B. McKenna, J. Non-Cryst. Solids 172-174, 756 (1994).

[28] A. Q. Tool, J. Am. Ceram. Soc. 29, 240 (1946).

[29] S. Rekhson, J. Non-Cryst. Solids 95-96, 131 (1987).

[30] O. S. Narayanaswamy, J. Am. Ceram. Soc. 54, 491 (1971).

[31] C. T. Moynihan et al., Ann. N.Y. Acad. Sci. 279, 15 (1976).

[32] I. M. Hodge, J. Non-Cryst. Solids 169, 211 (1994).

[33] J. Mijovic, L. Nicolais, A. D’Amore, and J. M. Kenny, Polym. Eng. Sci. 34, 381 (1994).

[34] I. Avramov, Thermochim. Acta 280-281, 363 (1996).

[35] A. Alegria, L. Goitiandia, I. Tellerian, and J. Colmenero, Macromolecules 30, 3881 (1997).

[36] A. J. Kovacs, Adv. Polym. Sci. 3, 394 (1963).

[37] G. W. Scherer, J. Am. Ceram. Soc. 67, 504 (1984). 